\title{
Problemáticas fundamentales de la formación en física básica
}

\author{
César Alexander Chacón Cardona ${ }^{1}$ \\ Artículo presentado el 4 de agosto de 2008 y aprobado el 27 de noviembre de 2008. \\ Fundamental problems in basics physics \\ formation
}

\begin{abstract}
Resumen: En las labores docentes de los profesores universitarios, no es un secreto la existencia de un bajo desempeño y alta mortalidad académica de los estudiantes de física de las diversas carreras (ciencias naturales, ingenierías, ciencias de la educación, tecnologías, etc.) que cuentan en sus programas curriculares con esta disciplina como base de su formación. En el trabajo desarrollado a continuación se exponen algunos de los problemas más sobresalientes de la enseñanza de la física y se propone el desarrollo de la física computacional como estrategia útil para el desarrollo de aprendizaje significativo de esta ciencia en estudiantes de primeros semestres del nivel universitario.
\end{abstract}

Palabras clave: Mortalidad académica, enseñanza, aprendizaje, física computacional.
Abstract:Inside the educational job in the academic spaces, there is no secret about the low level of performance and the high mortality of the physics students from several programs (natural sciences, engineering, educational sciences, technologies, etc.) that count with this discipline like a basis of formation. In this paper the most important problems in the teaching of physics are exposed and the use of computational physics is proposed like useful tool in the science's meaningful learning for the new students of university level.

Key words: Academic decease, teaching, learning, computational physics.

1 Universidad Distrital Francisco José de Caldas, Bogotá. alexchacord@yahoo.com 


\section{Presentación}

Los resultados de la enseñanza de la física en los primeros semestres universitarios no son muy diferentes a los que enfrentan los docentes de los cursos de física de la enseñanza escolar, en la cual la percepción de los estudiantes acerca de esta ciencia es negativa, aun antes de iniciar su estudio (Benegas, 2007).

Por esta razón, diferentes grupos de investigación en docencia han estado trabajando intensamente en la determinación de causas de los deficientes desempeños académicos en los estudiantes de física, cuya mortalidad académica ha sido evaluada en algunos casos con índices superiores al cincuenta por ciento (Cravino y Lopes, 2003). La práctica docente en nuestro país no se escapa a estos niveles elevados de mortalidad, encontrando falencias fundamentales que impiden un aprendizaje significativo de la física, problemáticas que conllevan a plantear un refuerzo de los procesos de aprender a aprender, de saber hacer, de educabilidad, de aplicabilidad y de enseñabilidad de los estudiantes de primeros semestres de enseñanza básica universitaria. Estas problemáticas centradas en el hacer del maestro son:

1. La falta de una didáctica acorde con la naturaleza de la física que facilite y optimice los procesos de enseñanzaaprendizaje significativos de las bases conceptuales.

2. La descontextualización del conocimiento adquirido respecto al marco histórico y al entorno de aplicación de los saberes aprendidos.

3. Las fallas reiterativas en el planteamiento y solución de problemas.

\section{Conceptualización en física}

Las dificultades de aprendizaje significativo de conceptos son un problema esencial de la didáctica de la física. El desempeño académico de los estudiantes depende de la formación de modelos mentales basados en los conceptos, proposiciones (formulaciones matemáticas, definiciones) analogías y procedimientos experimentales de la física (Greca y Moreira, 1998). Es por esta razón que la comunicación de esta disciplina desde los docentes hacia los estudiantes debe evitar convertirse en una exposición indiscriminada de fórmulas y ecuaciones sin ningún tipo de comprensión del significado físico contenido en cada proceso simbólico (Pontes y De Pro, 2001). Las ideas intuitivas de los estudiantes de primeros semestres, el contexto socio-económico de los aspirantes, la formación científica de los docentes, así como las diferencias cualitativas de enseñanza-aprendizaje entre los niveles de bachillerato y universitario, determinan el grado de formación de los futuros profesionales. (Cravino y Lopes, 2003).

La mayoría de los expertos consideran que los conocimientos previos a la formación escolar, fruto de las experiencias cotidianas de los jóvenes, comenzando con las ideas extraídas desde los espacios lúdicos y las interrelaciones sociales con sus familiares y sus pares, son determinantes de la apropiación posterior del conocimiento. Estos preconceptos comunes a estudiantes de diferentes medios y edades están dotados de una estructura coherente, no son ajenos a las concepciones que estuvieron vigentes larguen el transcurso de la historia y no se modifican fácilmente 
por medio de la enseñanza tradicional, estando fuertemente ligados.

Los estudiantes contestan, de una forma relativamente correcta, al tipo de evaluaciones teóricas empleadas con frecuencia en los exámenes. Al introducir otro tipo de preguntas en las que se requiere más que la exposición de la información, un análisis de situaciones en el cual sea necesario sugerir soluciones más allá de los ejemplos tipo, se evidencia una grave y general incomprensión de los conceptos más fundamentales y reiteradamente enseñados. Por ejemplo, la pregunta cualitativa ¿qué experimentos se puede realizar para distinguir un ascensor opaco en reposo de uno igualmente opaco, moviéndose con velocidad constante? Muestra que un porcentaje muy alto de estudiantes universitarios considera que puede distinguir a nivel experimental los sistemas de referencia inercial. Y ello, después de haber resuelto decenas de ejercicios numéricos sobre las Leyes de Newton, y aún en clases de relatividad en la Física Moderna. (Berenguer y Selles, 2000)

Por eso, una gran mayoría de los estudiantes afectados en sus cimientos cognoscitivos no logran articular su base conceptual y, por tanto, carecen de una estructura sólida que les garantice un desempeño de alta calidad a futuro. Esta situación conduce a un círculo vicioso, los docentes con concepciones inestables ponen fundamentos de igual naturaleza en futuros estudiantes universitarios, llevando a que el estudio de la física a este nivel se convierta en una experiencia traumática en medio de la cual los estudiantes que no se adapten rápidamente al ritmo de estudio, pasan a engrosar las filas de la deserción académica. En esta situación, lo más difícil no es edificar conocimiento científico sobre bases insuficientes, sino edificar sobre bases conceptuales erróneas, ya que la labor docente debe primero centrarse en derribar falsas premisas que impiden la comprensión, para luego volver a fundamentar conceptos universalmente aceptados, que debían haberse establecido con anterioridad (Hernández, 2004).

\section{Contextualización en física}

Otro de los grandes problemas de la enseñanza-aprendizaje de la física es la poca relación del conocimiento adquirido con el entorno en el que dichos conceptos fueron desarrollados, así como su interiorización y aplicación a la cotidianidad. En primer lugar, la relación entre el entorno histórico y el desarrollo del conocimiento es opacada por la operatividad matemática, perdiéndose en el camino el significado primario de los conceptos. El docente muchas veces no llega a trascender hacia todo el aprendizaje significativo físico y filosófico de las concepciones; cómo se desarrollan, evalúan y cambian las teorías científicas, los orígenes de los conceptos, así como su aplicabilidad al entorno (Dapía, 2008).

Uno de los problemas de contexto más sobresalientes en la enseñanza de la física es la ausencia de conexión histórica en el desarrollo de las asignaturas propias de esta rama del pensamiento científico. Se enfocan los contenidos propios de cada área a la formulación sistemática de contenidos, sin reconocer que la ciencia necesita para su interpretación el análisis del marco histórico que ha permitido su desarrollo, avance y consolidación, trayendo como consecuencia la introducción de tergiversaciones y errores históricos. El resultado de esta 
hermenéutica deficiente hace que los estudiantes tiendan a tomar una imagen deformada de la manera en que se construyen y evolucionan los conceptos científicos, ignorando las situaciones problemáticas que originan las investigaciones y que pueden producir cambios en las teorías existentes. Se enfatiza como principal motivo para la creación teórica la elegancia matemática, así como que la física viene como resultado del esfuerzo de genios aislados (Galileo, Newton, Maxwell, Einstein, es decir, los héroes del Salón de la Física), olvidando que es fruto del trabajo de muchos hombres, enmarcado este esfuerzo en un contexto social determinante de desarrollos y retrocesos. Finalmente, no se muestra el carácter tentativo de la ciencia, los errores que aparecen en el proceso de creación que generan las nuevas ideas científicas y aun los resultados infructuosos en la búsqueda del conocimiento (Solbes y Traver, 1996).

Además de la deficiente contextualización con el entorno, existe este mismo problema en la interacción teoría-práctica en las experiencias de laboratorio. Siempre se está intentando comprobar la validez teórica con el experimento, pero no se concibe como parte fundamental del desarrollo del conocimiento, se aísla en un compartimiento mental la física experimental, separándola de la física teórica, siendo imposible comprender la una sin la otra. No se enfatiza la importancia de lo cotidiano en las clases de ciencias con el fin de mejorar las actitudes de los alumnos hacia esta ciencia (Aragón Méndez, 2004).

No se muestran las múltiples opciones de aplicabilidad de la física a problemas concretos del entorno o del país, ya que nuestros estudiantes presentan una dicotomía muy fuerte entre el aprendizaje y la aplicabilidad del conocimiento fuera del aula. Es necesario utilizar contextos de enseñanza-aprendizaje que despierten el interés de los estudiantes, para que se involucren en la comprensión de problemas próximos a sus vidas, su ciudad y su nación (Cravino y Lopes, 2003).

\section{Planteamiento y solución de problemas en física}

En la enseñanza tradicional de la física, el lugar que tiene el planteamiento y solución de problemas es de carácter fundamental. Desde la mecánica newtoniana hasta la mecánica cuántica, la transmisión de los conocimientos por el profesor en su estado final (decir lo que es, o explicar cómo se hace, de una manera directa y acabada, junto con la realización de ejercicios) se presenta como parte de la didáctica de la física (Becerra-Labra, Gras-Martí y MartínezTorregrosa, 2007). En cada texto orientado a la enseñanza-aprendizaje de esta disciplina, las secciones dedicadas a problemas ejemplo, problemas propuestos (ejercicios) y estrategias de solución ocupan un lugar prominente en los temas. Aún recordamos las palabras de Feynman, quien elocuentemente afirmó: "Usted no sabe nada sobre algo hasta que lo ha practicado" (Serway, 1996). Esta preponderancia ha llevado al abuso en la solución de ejercicios, convirtiéndose en la práctica en un factor que incentiva al aprendizaje memorístico. En lugar de actuar basados en razonamientos coherentes, los estudiantes memorizan procedimientos de resolución sin entender correctamente la estrategia utilizada, desarrollan una serie de problemas similares, pero son incapaces de utilizar 
dicha estrategia en un contexto distinto. Este mismo proceso memorístico, unido con una fundamentación conceptual limitada, determina que el estudiante no tenga las herramientas suficientes para interpretar sus resultados, verificar sus respuestas y entender sus alcances (Cravino y Lopes, 2003).

Las consecuencias de las tres problemáticas se resumen en el siguiente diagrama en el que se muestran las ramificaciones no sólo en el ambiente académico, sino también en el desempeño profesional de los egresados, ya sea como profesionales en las diversas ramas del conocimiento que tienen en la física su fundamento (ciencias naturales, ingeniería, y afines) como docentes universitarios, como investigadores, así como la baja preparación en posgrado a la que pueden tener acceso en el futuro.

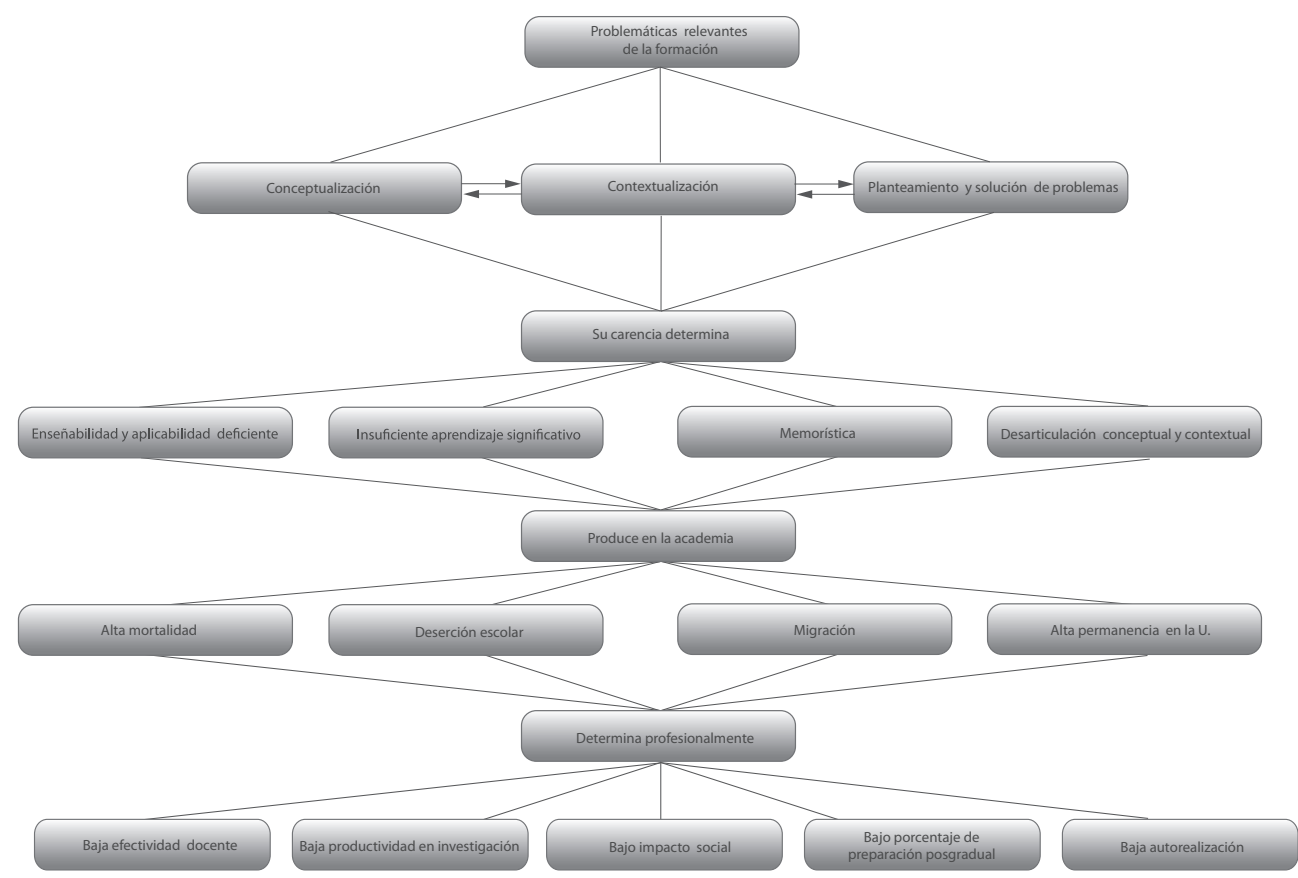

Figura 1: Ramificaciones de las problemáticas en la formación en física básica.

\section{Enseñanza de la física por medio de herramientas computacionales}

Las dificultades de conceptualización, contextualización y resolución de problemas están íntimamente relacionadas, se alimentan una a la otra, y la solución de una involucra necesariamente la de las otras. Es aquí cuando la pedagogía cobra sentido, en la búsqueda de la aplicación de una didáctica acorde con la naturaleza de la física. La didáctica, considerada en la antigüedad como el arte de enseñar, hoy es, además, entendida como ciencia encaminada a orientar, 
dinamizar y optimizar los procesos de enseñanza-aprendizaje, que comprometen al docente y al estudiante en la difícil tarea de la formación integral del hombre. Ésta propicia una formación integral que permite que los conocimientos asimilados por los estudiantes tengan sentido en su formación científica (Ruiz, Martínez y Álvarez, 2006).

La enseñanza es la acción de proveer circunstancias para que el estudiante aprenda de manera significativa. El aprendizaje es significativo para el estudiante cuando éste encuentra sentido, significado y comprensión de los conocimientos. Por esta razón, en este trabajo se propone la investigación como estrategia fundamental y didáctica para el desarrollo de la docencia de la física, entendiendo la investigación como el proceso mediante el cual se desarrollan capacidades relacionadas con el reconocimiento de cuestiones clave, el levantamiento de información adecuada, la interpretación de datos, la evaluación de las pruebas, pues algunas fuentes son más fiables que otras, y la evaluación de puntos de vista contrarios, así como la comunicación y defensa de los propios (Acevedo, 2004). La investigación permite el ejercicio sistemático de búsqueda, reflexión y análisis de procesos educativos en la construcción de nuevos conocimientos y de nuevas formas de mirar el trabajo académico universitario (Díaz-Barriga y Hernández, 2001).

Aunque la propuesta de investigación está enfocada en primera instancia a corregir las debilidades observadas en la resolución de problemas físicos, las otras dos problemáticas no serán dejadas de lado. Si bien en la sección anterior se describieron cada uno de los proble- mas por separado, es necesario anotar que detrás de ellos existe una causa primordial: la enseñanza de la física dependerá en gran medida de la habilidad y destreza para la enseñanza-aprendizaje en los estudiantes del arte de aprender a aprender. En esta didáctica, debe ser claro que no es posible entender los conceptos de la física si no se ha tenido un proceso de asimilación, interiorización y comprensión de ellos, no es posible aplicar dicho conocimiento a situaciones reales si no se ha logrado mediante la integración de diferentes áreas de conocimiento desarrollar habilidades, un pensamiento sistémico, estructurado, lógico y formal, y además no es posible plantear ni resolver problemas complejos ni crear nuevo conocimiento, si no se han desarrollado los procesos básicos de aprendizaje significativo autónomo (Buzán y Buzán, 1996).

De esta manera, el planteamiento y solución de problemas en física será desplegado por medio del desarrollo de investigación en la enseñanza-aprendizaje utilizando herramientas propias de la física computacional que posibiliten una didáctica para el aprendizaje de la física. En las últimas dos décadas, esta disciplina se ha convertido en un campo de estudio obligado, debido a que ofrece al estudiante la habilidad de realizar cálculos y experimentos numéricos con las ayudas computacionales existentes, compitiendo en importancia con áreas de enseñanza tradicionales, como la física teórica y la física experimental (Estrada, 2004).

Mediante la enseñanza de la física computacional es posible complementar la formación tradicional de los profesionales, aplicando los análisis de 
fenómenos naturales a problemas más cercanos a la realidad, no sólo con el desarrollo de soluciones analíticas, sino aproximándose a ellas por medio de técnicas numéricas. Además, teniendo en cuenta el contexto tecnológico actual, el desarrollo de las herramientas computacionales permite abordar problemas experimentales de gran complejidad a un costo relativamente bajo, logrando así la aplicación de los fundamentos aprendidos, la interacción del estudiante con su entorno y la solución de problemas abiertos (Rosado y Herreros, 2005). Para lograr el cumplimiento de estos fines se plantea a continuación la siguiente metodología:

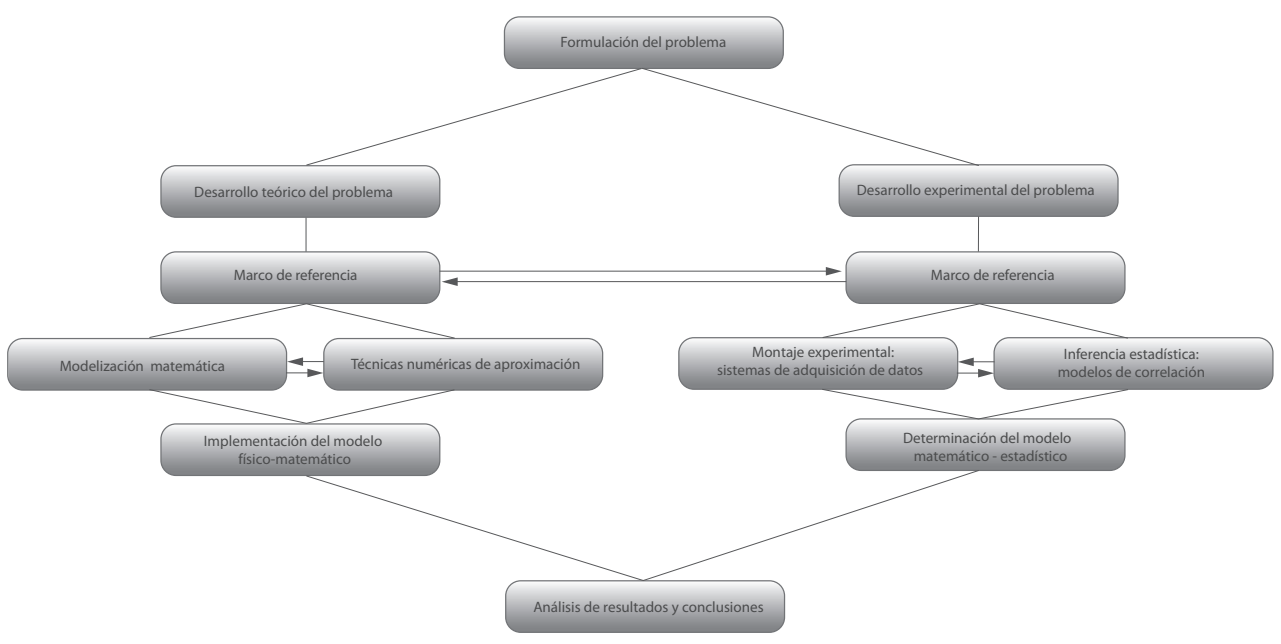

Figura 2: Metodología para el desarrollo de la física computacional.

La solución de problemas por medio de la física computacional puede ser orientada principalmente en dos campos, modelización matemática del problema e inferencia estadística de modelos de correlación con información proveniente desarrollos experimentales propuestos para el mismo problema. En la primera sección de estos dos grupos se buscará, por medio de un seminario introductorio, el planteamiento de problemas reales basados en la formación básica del estudiante con el fin de es- bozar propuestas de investigación que puedan encaminarse y complementarse en las dos secciones. La primera parte contiene el desarrollo del proyecto, partiendo de un proceso de contextualización en el marco de referencia, continuando con el análisis de los modelos físico-matemáticos y las técnicas numéricas de aproximación adecuadas al problema teórico (Silva y Tello, 2006), mientras que en el caso experimental se profundizará en el desarrollo de instrumentación, los sistemas de adquisición 
de datos, el tratamiento estadístico de la información y los modelos de correlación susceptibles de utilizar. Al final de este proceso, el estudiante estará en la capacidad de implementar un modelo adecuado para su problema en particular y solucionarlo evaluando los resultados y sus implicaciones para finalmente comparar las predicciones modeladas computacionalmente con el análisis de resultados proveniente del desarrollo experimental (Castellanos y D’Alessandro, 2003).

Se propone utilizar como estrategia docente para un aprendizaje significativo el desarrollo de las asignaturas de física básica conducentes al perfeccionamiento de trabajos de grado con énfasis en la utilización de herramientas computacionales. Aquí, el docente tomará el lugar de facilitador del proceso de aprendizaje de los estudiantes, llevándolos a que sean protagonistas de su formación.

Por medio de la interacción de los estudiantes y el docente en el planteamiento del problema, se pretende desarrollar la capacidad del estudiante de obtener información por sí mismo, contextualizar la ciencia a problemas de su entorno y enfocar sus esfuerzos en la comprensión y planteamiento de problemas concretos. Una vez elegido el problema a resolver y habiendo delimitado su propuesta, se buscará encaminar al estudiante en la solución del problema específico por medio de tareas dirigidas, escudriñando siempre la aplicación de bases conceptuales previas, fundamentos matemáticos y numéricos, así como la utilización de la tecnología en el quehacer docente. De esta forma, se pretende que la práctica de la física en situaciones concretas genere un conocimiento didáctico que contemple tanto los principios y explicaciones de los fenómenos como los procedimientos y aplicaciones de dicho conocimiento. La enseñanza-aprendizaje de la física por medio de la computación se vuelve más interactiva, enfocándose no sólo en la transmisión-recepción de información, sino proporcionando un ambiente en el que la ciencia se convierta en una experiencia vivencial y no simplemente académica.

Como docente universitario, el anhelo más ferviente es formar nuevas generaciones no sólo en el aprendizaje de la física, sino en la aplicación de esta disciplina científica a la solución de problemas de la vida diaria. Cuando se logra esto, interactuactuando con los estudiantes, sacándolos de su ensimismamiento conceptual, afirmándolos en bases sólidas de aprendizaje y llevándolos al punto en que puedan pensar autónomamente en una forma coherente, puede decirse que se ha cumplido la misión.

Con el paso de los años, se aprende que la física es todo un arte, se forma por medio del hacer, no simplemente a través de esquemas facilistas de memorización o solucionando unos cuantos ejemplos. Se aprende como parte de la vida, de manera que cada docente de esta área del conocimiento tiene la responsabilidad de transmitir precisamente sus bases conceptuales, demostrar la manera en que el entorno afecta y es afectado por la ciencia, y cómo se puede interactuar con la naturaleza para el provecho del ser humano.

Es posible interesar a los estudiantes en la enseñanza-aprendizaje de la física, 
es posible disminuir la apatía y la mortalidad académica de esta disciplina, si se parte del desarrollo de las capacidades de aprendizaje significativo autónomo. La física es una disciplina que propicia el desarrollo del olvidado arte de pensar, y los docentes son llamados a facilitar esta labor proveyendo a cada discípulo las herramientas y estrategias pedagógicas adecuadas a sus necesidades e intereses.

Con la aplicación de la física por medio de herramientas computacionales,

\section{Referencias}

Acevedo, J. A. (2004). Reflexiones sobre las finalidades de la enseñanza de las ciencias: educación científica para la ciudadanía. Eureka sobre Enseñanza y Divulgación de las Ciencias, 1(1), 3-16

Aragón, M. (2004). La ciencia de lo cotidiano. Eureka sobre Enseñanza y Divulgación de las Ciencias, 2(1), 109-121

Benegas, J. (2007). Tutoriales para física introductoria: una experiencia exitosa de aprendizaje activo de la física. Latin-American Journal of Physics Education, 1(1).

Becerra-Labra, Gras-Martí y MartínezTorregrosa. (2007). La física con una estructura problematizada: efectos sobre el aprendizaje conceptual, las actitudes e intereses de los estudiantes universitarios Revista Brasileira de Enseñanza de la Física, 1(29).

Berenguer, R. y Selles, J. (2000). Enseñanza por cambio conceptual: de la física clásica a la relatividad. Enseñanza de las Ciencias, 18(3), 463-471.

Buzán, T. y Buzán, B. (1996). El libro de los mapas mentales. Barcelona: Ediciones Urano. los jóvenes llegarán a afirmar sus bases conceptuales recibidas desde sus tempranas etapas de formación; mediante la contextualización de su aprendizaje podrán articular estas bases como un conocimiento estructurado y útil para la aplicación de la ciencia en la solución de problemas concretos, desarrollando durante este proceso las disciplinas propias del investigador científico y la capacidad de aplicar el conocimiento a nuestra sociedad.

Cravino, J., Lopes, B. (2003). La enseñanza de la física general en la universidad. Propuesta de investigación. Enseñanza de las Ciencias, 21(3), 473-482.

Castellanos, M. y D'Alessandro, A. (2003). Proyectos de investigación: una metodología para el aprendizaje significativo de la física en educación media. Revista Pedagógica, 69(24), 101-136.

Dapía, A. (2008). Deconstrucción de la didáctica racionalista en el contexto de la formación docente. Hacia una didáctica constructivista. Revista Iberoamericana de Educación, 45, 3-25.

Díaz-Barriga, F. y Hernández, G. (2001). Estrategias docentes para un aprendizaje significativo. México: McGraw-Hill.

Estrada, H. (2004). Física computacional. Bogotá: Universidad Nacional de Colombia-Facultad de Ciencias.

Greca, I. y Moreira, M. (1998). Modelos mentales y aprendizaje de física en electricidad y magnetismo. Enseñanza de las Ciencias, 16(2), 289-303.

Hernández, C. (2004) Física para diseñadores industriales: ¿qué y cómo aprenden cuando diseñan? Revista de Estudios Sociales, 19, 15-31. 
Rosado, L. y Herreros, J. (2005). Nuevas aportaciones didácticas de los laboratorios virtuales y remotos en la enseñanza de la física. Recente Research Development in Learning Technologies, Formates, 1-5.

Pontes, A. y De Pro, A. (2001). Concepciones y razonamientos de expertos y aprendices sobre electrocinética: consecuencias para la enseñanza y la formación de profesores. Enseñanza de las Ciencias, 19(1), 103-121.

Ruiz Mendoza, J., Martínez, H. y Álvarez, N. (2006). Estrategia didáctica para la formación integral del estudiante de bachillerato mediante el proceso de enseñaza-aprendizaje de la física.
Revista Iberoamericana de Educación, 40, 2-10.

Serway, A. (1996). Física. Mexico: Mc-Graw Hill.

Silva, M. y Tello, J. (2006). Estudio sobre el aporte efectivo del sofware Modellus durante el desarrollo de la metodología de modelamiento mental de Hestenes, para el aprendizaje de la física. Tesis para optar al Grado de Magíster en Educación, Facultad de Ciencias Sociales, Departamento de Educación, Santiago de Chile.

Solbes, J. y Traver, M. J. (1996). La utilización de la historia de las ciencias en la enseñanza de la física y la química. Enseñanza de las Ciencias, 14 (l), 103-112. 